\title{
Third Mobile Accessibility Workshop
}

Tiago Guerreiro

Faculdade de Ciências

University of Lisbon

Edifício C6, Piso 3.

Campo Grande,

1749-016 Lisboa, Portugal

tjvg@di.fc.ul.pt

\section{Jeffrey Bigham}

720 Computer Studies Building

University of Rochester

Rochester, NY 14627, USA

jbigham@cs.rochester.edu

\section{Luis Carriço}

Faculdade de Ciências

University of Lisbon

Edifício C6, Piso 3.

Campo Grande,

1749-016 Lisboa, Portugal

Imc@di.fc.ul.pt

\section{Daniel Gonçalves}

Instituto Superior Técnico

TULisbon

Rua Alves Redol 9,

1000-029 Lisboa, Portugal

daniel.goncalves@inesc-id.pt

\section{Yeliz Yesilada}

Middle East Technical University,

Northern Cyprus Campus,

Kalkanlı, Güzelyurt, TRNC,

Mersin 10, Turkey

yyeliz@metu.edu.tr

\section{Shadi Abou-Zhara}

World Wide Web Consortium

2004, Route des Lucioles B.P. 93

06902 Sophia-Antipolis, France

shadi@w3c.org

\begin{abstract}
Mobile interaction presents challenges that go beyond the traditional desktop contexts. These difficulties become even greater when considering people with disabilities or people without disabilities that experience situational impairments. Further, mobile devices and interfaces have been evolving at an astonishing rate which leads to increasing difficulties in maturing the field and consequently to ensure accessibility by different people and under different circumstances. This workshop aims to bring together researchers from the Mobile HCI, Mobile Accessibility, and Mobile Web areas, towards identifying the most prominent challenges for mobile accessibility and guidelines for a more structured and faster development of accessible solutions.
\end{abstract}

\section{Author Keywords}

Mobile Devices, Mobile HCI, Accessibility, Mobile Accessibility, Mobile Web, Situational Impairments.

\section{ACM Classification Keywords}

H5.m. Information interfaces and presentation (e.g., $\mathrm{HCI}$ ): Miscellaneous.

\section{General Terms}

Design, Experimentation, Human Factors.

\section{Introduction}

Mobile devices play an important role in current society. They are increasingly seen as extensions of one's body 
[1] and empower the user in several different ways, from leisure to productivity and both synchronous and asynchronous communication.

Given this relevance and the pervasive usage of mobile devices by one's social and professional networks, the inability to effectively operate such a tool is likely to be a strong vehicle of exclusion. Counteracting its initial purpose of improving communication among people anytime and anywhere, the widespread but noninclusive usage of mobile devices is likely to widen the gap between disabled and non-disabled people.

Conversely, if access to these tools is provided they can be empowering to disabled people improving their communication, productivity, cultural and leisure opportunities. In the overall, they can improve one's independent living [2].

Mobile devices are increasingly visual, making them hard to use by blind or vision-impaired people.

Keyboards are steadily being replaced by touchscreens, without tactile feedback, introducing additional barriers. Tetraplegic users have limited mobility of their limbs and hardly are able to pick up the devices or use their fingers for precise pointing [3].

Paradigm shifts have been occurring not only in the way people interact with the devices but also on the contents and environments mobile interaction deals with. Mobile Web is a reality and the challenges posed by its inherent complexity are exponentially higher when considering disabled or situationally-impaired people [4]. Indeed, the mobile aspect often creates situations where those interaction modes are cumbersome or socially unacceptable, even for non- impaired people. Overall, the list of challenges is immense.

Fortunately, there is a nascent area of research, Mobile Accessibility, where researchers are focusing on solving accessibility problems in mobile devices and applications. Bringing together Mobile $\mathrm{HCI}$ and Accessibility, it is the source of synergistic works that have the potential of deeply transforming how we look at mobile devices, and to shape the ongoing mobile revolution. Work in this area ranges from new text introduction techniques to multimodal interaction solutions, mobile assistive technology, prototyping tools, navigation tools, theoretical models, etc. A wide range of users and situations are also considered (blind or situationally blind, motor-impaired or people with movement-induced impairments, elderly, deaf, etc.). As a result, solutions provided to people to disabilities could be applied to the aforementioned situationally impaired users.

This workshop will focus on identifying future opportunities for improving mobile accessibility for both disabled and situationally-impaired users. Particularly, it will focus on identifying how to reduce the time between device and interface breakthroughs and the deployment of their accessible versions. $\mathrm{CHI}$ is especially suited to host this workshop as it is the main forum bringing together researchers from the mobile $\mathrm{HCI}$ and Accessibility community and where major interface breakthroughs are presented and studied. 


\section{Workshop Goals}

One of the main goals of this workshop is to create a cross-disciplinary platform to bring together

researchers and practitioners interested in designing or improving the accessibility of mobile interactive

systems, environments and contents. In particular, we intend to bring together the accessibility, mobile

interaction, and mobile web communities. Papers on Mobile Accessibility have become an increasing presence in conferences such as CHI, ASSETS, W4A and MobileHCI which is a proof of a growing and active community. However, there has been a lack of a single well defined venue for the researchers in the area to meet, exchange ideas and results, and to advance the knowledge to support better inclusion.

\section{Additional workshop goals include:}

- Highlight research challenges for this community to guide future research;

- Identify abilities and situations that can be matched towards the globalization of mobile accessibility;

- Identify guidelines for researchers to reduce the delay of accessible interface counterparts;

- Discuss interfaces, standards and guidelines under development (e.g, in the web context, HTML 5), challenges, and their concordance with mobile accessibility

- $\quad$ Share best practices.

\section{Workshop Topics}

The workshop organization is composed of researchers with past experience on studying and designing mobile accessible interfaces, improving accessibility and quality of life by using mobile technologies, and designing and standardizing accessible interfaces and contents. Particularly, this edition of the workshop has united the valences and interests already stressed in the two previous editions (INTERACT 2011 and MobileHCI 2012), focusing mainly on input and output modalities and accessible applications, with the parallel area of Mobile Web Accessibility ${ }^{1}$, a relevant and current area of research, that is likely to spice the discussion before, during and after the workshop. Topics for the workshop include:

- Mobile Accessibility

- Mobile assistive technologies Dealing with Individual Differences Dealing with Situational Impairments

- Correlating User Disabilities and Situational Impairments

- The Disability Continuum and Adaptation

- Mobile Interaction Contexts and Scenarios

Empowerment through mobile devices Guidelines for Mobile Interface Design Mobile Web accessibility challenges

Mobile Web Standards and Guidelines

- Relationship between Mobile Web and Mobile Devices and Paradigms

HTML 5.0 Accessibility

- Evaluating Web Accessibility

${ }^{1}$ W3C/WAI Research and Development Working Group (RDWG)
Online Symposium on Mobile Accessibility http://www.w3.org/WAI/RD/2012/mobile/ 


\section{Workshop Format}

Discussion between participants around topics of the workshop will start long before the workshop, after the notification of acceptance given to the authors. The webpage of the event will soon display all accepted position papers and will comprise an area for discussion (wiki or forum) for each paper. Regularly, between the acceptance and the workshop day, we will send an email to the authors of all papers asking them to read and comment a given paper, fostering the discussion around all accepted papers. This will start the discussion long before the workshop itself and will help us identifying the most prominent topics for discussion.

At the workshop, we will start the day with presentations from the authors of the most discussed papers/topics. The remaining contributions will be presented in an interactive poster session where demos will be encouraged and discussion will be fostered. Following, we will perform round-table discussions focusing on the aforementioned workshop goals. The last part of the workshop will be based on the CHI 2013 main program itself, where the organization committee will present mobile inaccessible breakthroughs presented in this year's edition of the conference and each group will lean over the solution to come up with an accessible counterpart and on ideas on how that accessibility could be achieved (authors of those papers will be contacted for permission and for presence at the workshop)

The first two editions of the workshop contemplated extended versions of selected papers to a special issue of the Universal Access in the Information Society journal. We are undergoing negotiations to keep that tradition for this third edition.

\section{References}

[1] Townsend, A.. Life in the Real-Time City: Mobile Telephones and Urban Metabolism. Journal of Urban Technology, 7(2):85-104, 2000.

[2] Bigham, J.P., White, S., Yeh, T., et al. VizWiz: Nearly real-time answers to visual questions.

Proceedings of the 23nd annual ACM symposium on User interface software and technology - UIST '10, ACM Press (2010), 333.

[3] Guerreiro, T., Nicolau, H., Jorge, J., and Gonçalves, D.. Assessing mobile touch interfaces for tetraplegics. In Proceedings of the 12th international conference on Human computer interaction with mobile devices and services, MobileHCI '10, pages 31-34, New York, NY, USA, 2010. ACM.

[4] Yeliz Yesilada, Tianyi Chen, and Simon Harper. A simple solution: solution migration from disabled to small device context. In Proceedings of the 2010 International Cross Disciplinary Conference on Web Accessibility (W4A), W4A '10, pages 27:1-27:2, New York, NY, USA, 2010. ACM. 